\title{
Effects of microbial population in degradation process of xenobiotic polymers
}

\author{
M. Watanabe ${ }^{1} \quad$ F. Kawai ${ }^{2}$
}

(Received 3 March 2010; revised 30 August 2010)

\begin{abstract}
Microbial depolymerization processes of exogenous type are studied for biodegradation of polyethylene glycol. The time factor of a degradation rate is the microbial population which grows on degraded monomer units as the carbon source. Numerical simulation shows that our model is of practical use.
\end{abstract}

\section{Contents}

1 Introduction

C683

2 Modeling exogenous depolymerization processes

C684

3 Reduction to time averaged model

C685

http://anziamj . austms.org.au/ojs/index.php/ANZIAMJ/article/view/2433 gives this article, (c) Austral. Mathematical Soc. 2010. Published September 23, 2010. ISSN 1446-8735. (Print two pages per sheet of paper.) Copies of this article must not be made otherwise available on the internet; instead link directly to this URL for this article. 
4 Time factor of degradation rate based on microbial population

C687

5 Simulation of time transition of weight distribution and growth of microorganism

C690

6 Discussion

C694

References

C694

\section{Introduction}

Microbial depolymerization processes are classified into two types, exogenous type and endogenous type. In an exogenous depolymerization process, molecules lose their weight by separation of monomer units from their terminals. Examples of polymers subject to exogenous depolymerization processes include polyethylene (PE) and polyethylene glycol (PEG). The mechanism of PE biodegradation is based on two essential factors: the gradual weight loss of large molecules due to the $\beta$-oxidation and the direct consumption or absorption of small molecules by cells. A mathematical model based on those factors was proposed, and PE biodegradation was studied using the model $[1,9,2]$. Polyethylene glycol (PEG) is another example of polymer subject to exogenous depolymerization processes. The mathematical techniques originally developed for the PE biodegradation was extended to cover the biodegradation of PEG [4]. Problems were formulated to determine degradation rates based on the weight distribution of PEG with respect to molecular weight before and after cultivation of a microbial consortium E-1. Those problems were solved numerically, and the transition of the weight distribution was simulated. Dependence of degradation rate on time was also considered in modeling and simulation of depolymerization processes of PEG $[5,7,6,8]$. 
The study on dependence of degradation rate in time in depolymerization processes of PEG is continued. The time evolution of microbial population is taken into account in modeling of depolymezation process. Experimental data is introduced into analysis based on a model, and the transition of weight distribution is simulated.

\section{Modeling exogenous depolymerization processes}

In microbial depolymerization of PE, molecules lose weight gradually by separation of monomer units from terminals by ( $\beta$-oxidation) until they become small enough to be absorbed directly into cells. The PE biodegradation model is based on two essential factors, the gradual weight loss of large molecules due to $\beta$-oxidation and the direct consumption of small molecules by cells. Let $t$ and $M$ denote the time and the molecular weight respectively. A molecule with molecular weight $M$ is called an $M$-molecule. Let $L$ be the amount of weight loss due to the $\beta$-oxidation. Since a PE molecule loses two carbon units $\mathrm{CH}_{2} \mathrm{CH}_{2}, \mathrm{~L}=28$. Suppose that $w(t, M)$ represents the total weight of $M$-molecules present at time $t$. Suppose also that $\rho(M)$ represents the direct consumption rate, and that the function $\beta(M)$ represents the $\beta$-oxidation rate, which is the rate of the weight conversion from the class of $M$-molecules to the class of $(M-L)$-molecules due to the $\beta$-oxidation. Let $\alpha(M)=\rho(M)+\beta(M)$. The equation

$$
\frac{d x}{d t}=-\alpha(M) x+\beta(M+L) \frac{M}{M+L} y .
$$

was proposed as a model for PE biodegradation processes $[1,9,2,10]$. Here $x=$ $w(t, M)$, the total weight of $M$-molecules at time $t$, and $y=w(t, M+L)$, the total weight of $M+$ L-molecules at time $t$.

The mathematical model (1) is viewed as a general biodegradation model for exogenous depolymerization processes including biodegradaion of PEG. In 
an exogenous depolymerization process of PEG, a molecule is first oxidized at its terminal, and then an ether bond is cleaved. Since the monomer unit $\mathrm{CH}_{2} \mathrm{CH}_{2} \mathrm{O}$ is truncated in one cycle of depolymerization, $\mathrm{L}=44$ for exogenous depolymerization processes of PEG. The PEG molecules studied here are so large that they cannot be absorbed directly through membranes into cells. So $\rho(M)=0$, and Equation (1) becomes

$$
\frac{d x}{d t}=-\beta(M) x+\beta(M+L) \frac{M}{M+L} y .
$$

Equation (1) or (2) is adequate for depolymerization processes over a period in which the microbial population is stationary. However, the change of microbial population should be taken into account for a period in which it is developing or diminishing. Then the degradation rate is time dependent, and Equation (2) becomes

$$
\frac{d x}{d t}=-\beta(t, M) x+\beta(t, M+L) \frac{M}{M+L} y .
$$

Suppose that $f(M)$ is the initial weight distribution. Then Equation (3) is associated with the initial condition

$$
w(0, M)=f(M),
$$

and given a prescribed degradation rate $\beta(t, M)$, equation (3) and the initial condition (4) form an initial value problem.

\section{Reduction to time averaged model}

Time factors of depolymerization processes, such as microbial population, dissolved oxygen or temperature, affect molecules regardless of sizes because depolymerization processes are restricted to terminals. Then the degradation 
rate is separated into a time factor, which we denote by $\sigma(t)$, and molecular factor, which we denote by $\lambda(M)$, and the degradation rate is the product

$$
\beta(t, M)=\sigma(t) \lambda(M) .
$$

Then equation (3) becomes

$$
\frac{d x}{d t}=-\sigma(t) \lambda(M) x+\sigma(t) \lambda(M+L) \frac{M}{M+L} y .
$$

The time factor of the degradation rate is removed from the equation (6) by the time transformation

$$
\tau=\int_{0}^{t} \sigma(s) d s
$$

Let

$$
W(\tau, M)=w(t, M), \quad X=W(\tau, M), \quad Y=W(\tau, M+L) .
$$

Then

$$
\frac{d X}{d \tau}=\frac{d x}{d t} \frac{d t}{d \tau}=\frac{1}{\sigma(t)} \frac{d x}{d t},
$$

and equation (6) becomes

$$
\frac{d X}{d \tau}=-\lambda(M) X+\lambda(M+L) \frac{M}{M+L} Y .
$$

Given the initial weight distribution $f(M)$, equation (8) and the initial condition

$$
W(0, M)=f(M)
$$

form an initial value problem. Given an additional condition

$$
W(\mathcal{T}, M)=g(M),
$$

equation (8) and the conditions (9) and (10) form an inverse problem to determine the degradation rate $\lambda(M)$ for which the solution of the initial 
value problem (8) and (9) also satisfies the condition (10). When the solution $W(\tau, M)$ of the initial value problem (8) and (9) satisfies the condition (10), the solution $w(t, M)$ of the initial value problem (3) and (4) also satisfies

$$
w(T, M)=g(M),
$$

for which

$$
\mathcal{T}=\int_{0}^{T} \sigma(s) d s
$$

The inverse problem (8), (9) and (10) was solved numerically with techniques developed in previous studies. High performance liquid chromatography (HPLC) patterns were used in analysis as the weight distribution of PEG with respect to the molecular weight before and after cultivation of a microbial consortium E1 (Figure 1). Figures 2 shows the degradation rate $\lambda(M)$ based on the weight distribution before and after cultivation for three days $[6,8]$.

\section{Time factor of degradation rate based on microbial population}

The microbial population is the only time factor in the depolymerization process shown in Figure 1, and the monomer units separated from PEG molecules are the sole carbon sources. Then the time factor $\sigma(t)$ represents the microbial population, and the total amount of monomer units utilized by the microorganisms per unit time at time $t$ is

$$
A=\int_{0}^{\infty} \sigma(t) \lambda(M) w(t, M) d M .
$$

The microbial population increases under sufficient carbon supply when the ratio of the microbial population to the total amount $\sigma / A$ falls below a certain level $1 / h$ where $h$ is a positive constant, and it decreases under insufficient 


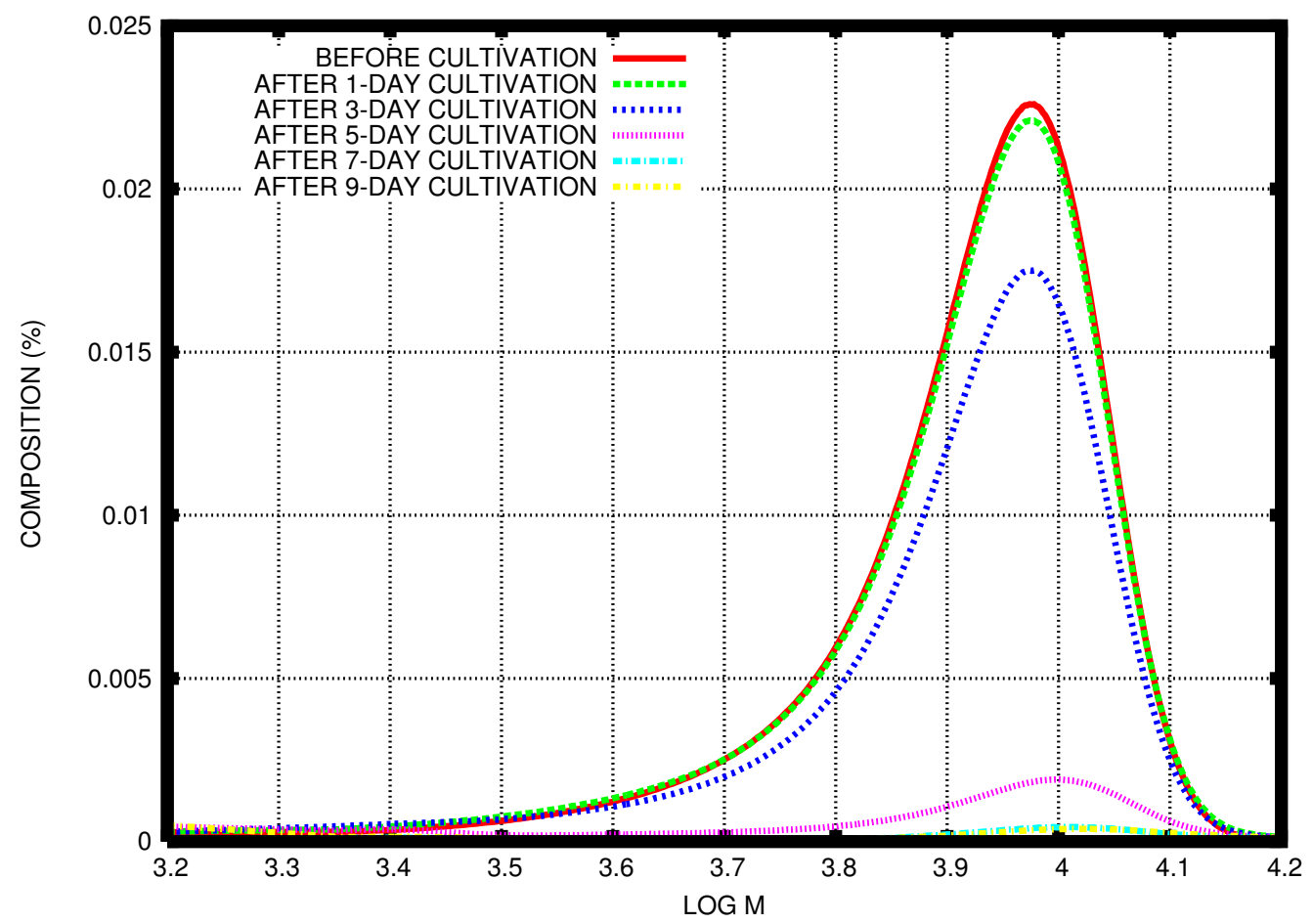

FiguRE 1: Weight distribution of PEG before and after cultivation of a microbial consortium E1 [6, 8]. 


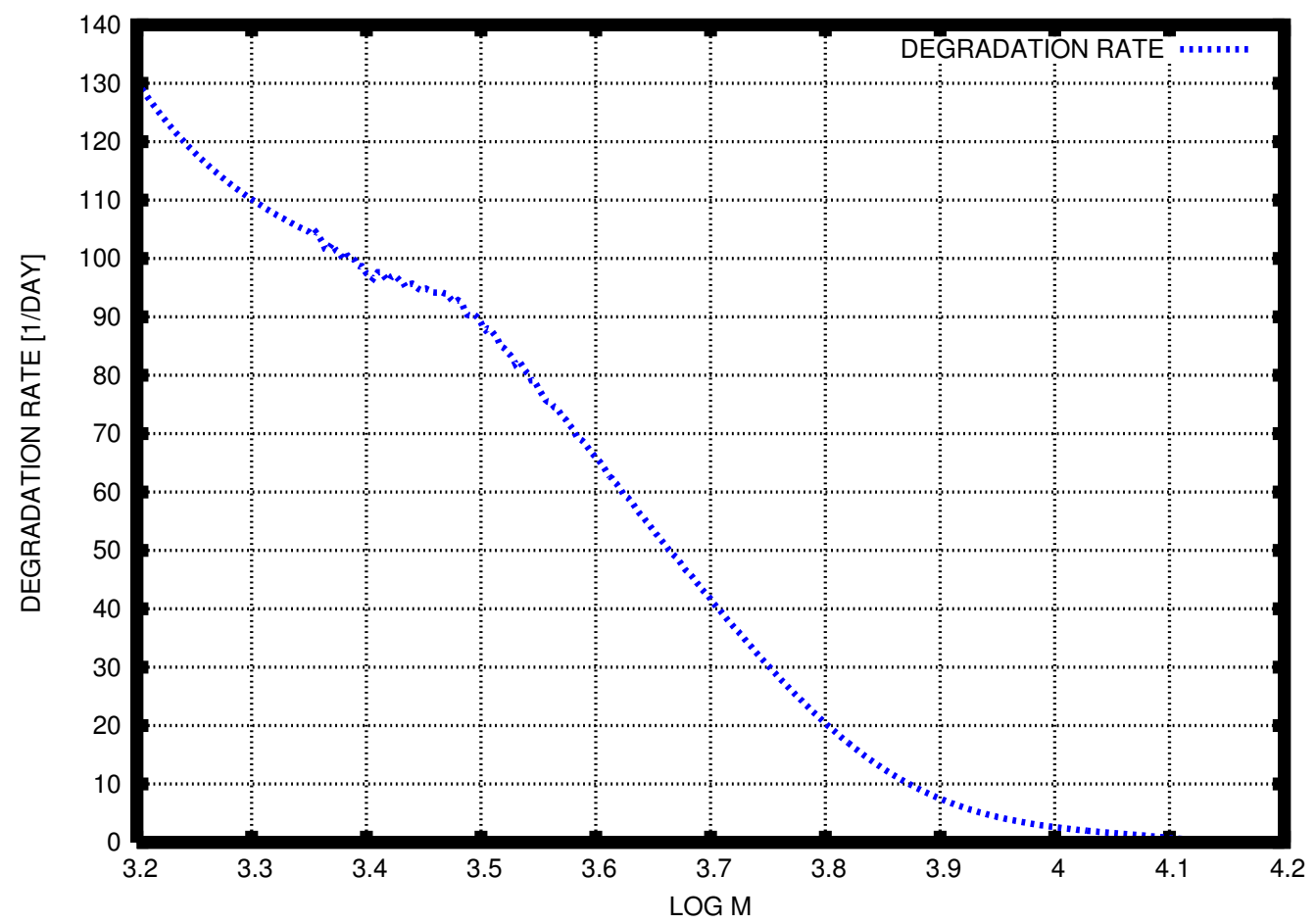

FiguRE 2: Degradation rate based on the weight distribution of PEG before and after cultivation of a microbial consortium E1 (Figure 1) [6, 8]. 
carbon supply when $\sigma / A$ exceeds $1 / h$. Then the growth rate of microbial population is proportional to

$$
1-\mathrm{h} \frac{\sigma}{\mathrm{A}}=1-\frac{\mathrm{h}}{\int_{0}^{\infty} \lambda(M) w(\mathrm{t}, M)},
$$

and the microbial population $\sigma(t)$ is a solution of the equation

$$
\frac{\mathrm{d} \sigma}{\mathrm{dt}}=\mathrm{k}\left(1-\mathrm{h} \frac{1}{\int_{0}^{\infty} \lambda(M) w(\mathrm{t}, M) \mathrm{dM}}\right) \sigma .
$$

Equation (13) is associated with the initial condition

$$
\sigma(0)=\sigma_{0}
$$

\section{Simulation of time transition of weight distribution and growth of microorganism}

Equations (3) and (13) are solved numerically to simulate the transition of weight distribution of PEG and growth of microbial population for $\sigma_{0} \approx$ $0.029827, k \approx 1.556758$, and $h=500.0$ [8]. Here those equations are solved numerically for $\sigma_{0} \approx 0.029827$, and $h=500.0$, and $k=1.9$ and $k=2.0$. Figures 3 and 4 show numerical results and experimental results for weight distribution after cultivation for three and five days, respectively. Figure 5 shows the corresponding time evolution of $\sigma(t)$.

The change of microbial population for the mixed culture E-1, S. terrae and Rhizobium sp. was investigated in a previous study of exogenous depolymerization process of PEG 6000 [3], where viable cell concentrations were determined by colony counting on nutrient agar plates. The population of viable $S$. terrae cells increased for the first three days under sufficient amount of residual PEG to sustain the growth, and then started decreasing rapidly after the consumption of PEG, which suggests that $S$. terrae plays 


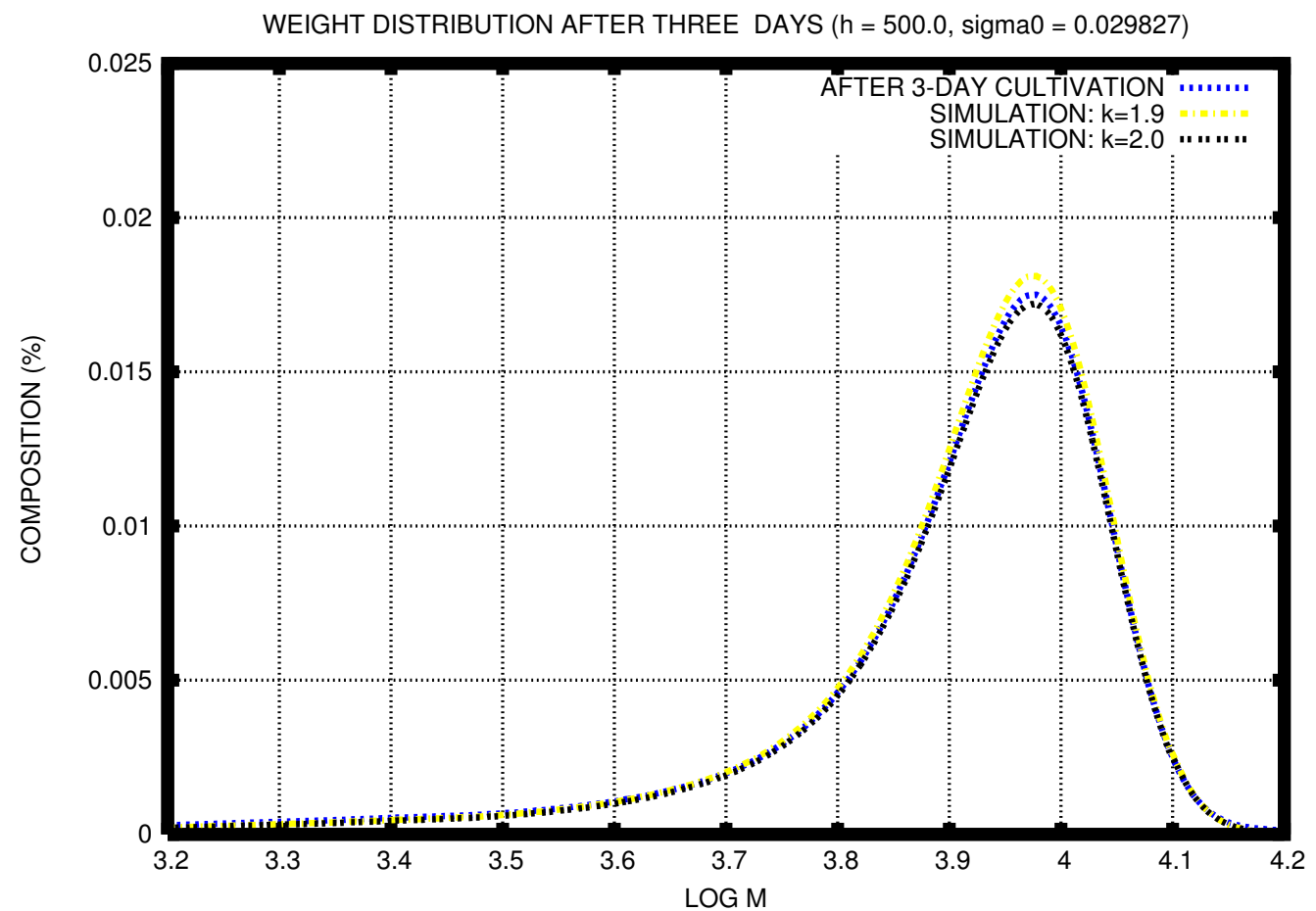

FiguRE 3: Numerical results and experimental result for weight distribution after three days of cultivation. $\sigma_{0} \approx 0.029827$ and $h=500.0$, and $k=1.9$ and $k=2.0$. 


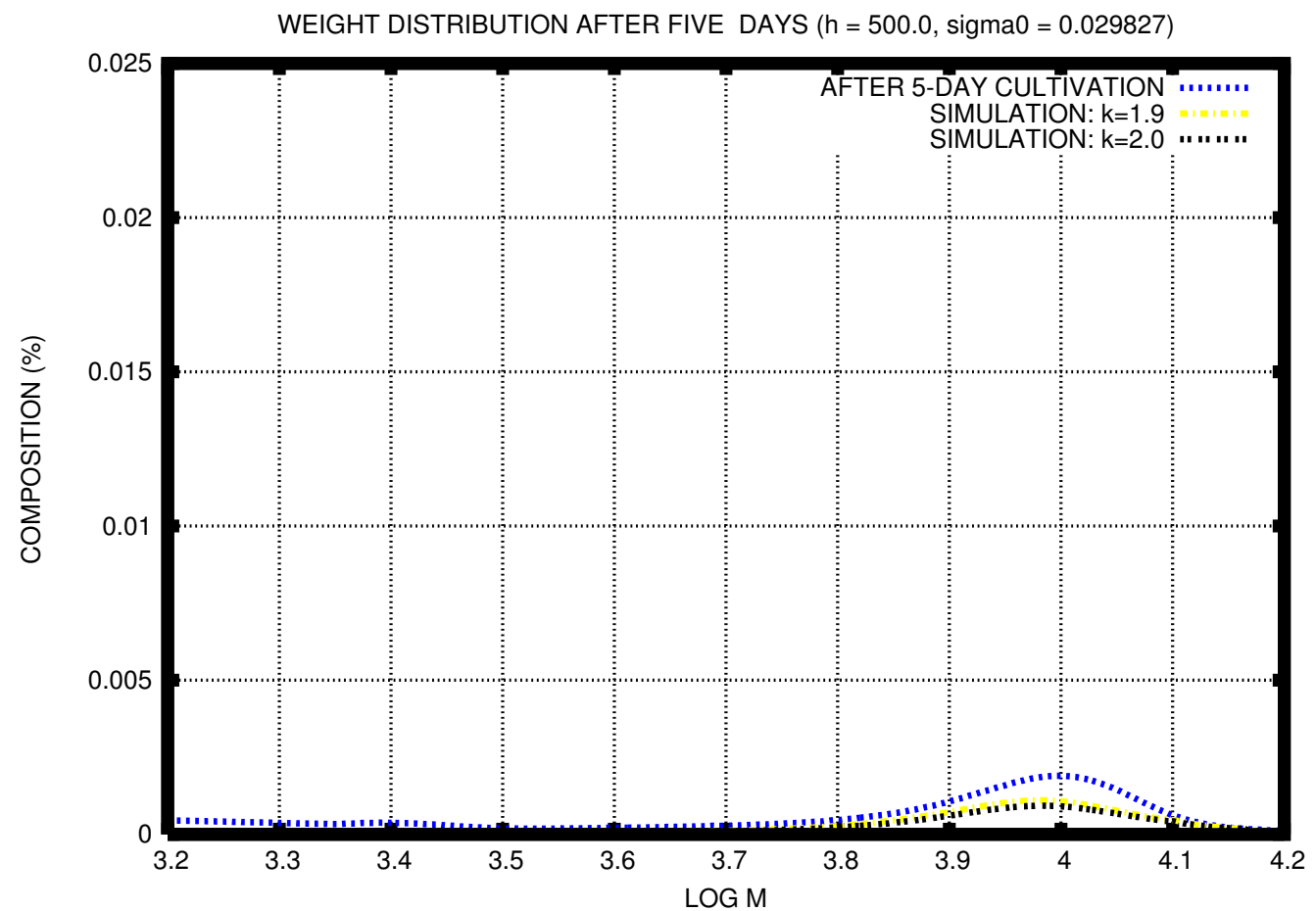

FiguRE 4: Numerical results and experimental result for weight distribution after five days of cultivation. $\sigma_{0} \approx 0.029827$ and $h=500.0$, and $k=1.9$ and $k=2.0$. 
5 Simulation of time transition of weight distribution and growth of microorganism(

Transition of microbial population $(h=500.0$, sigma0 $=0.029827$ )

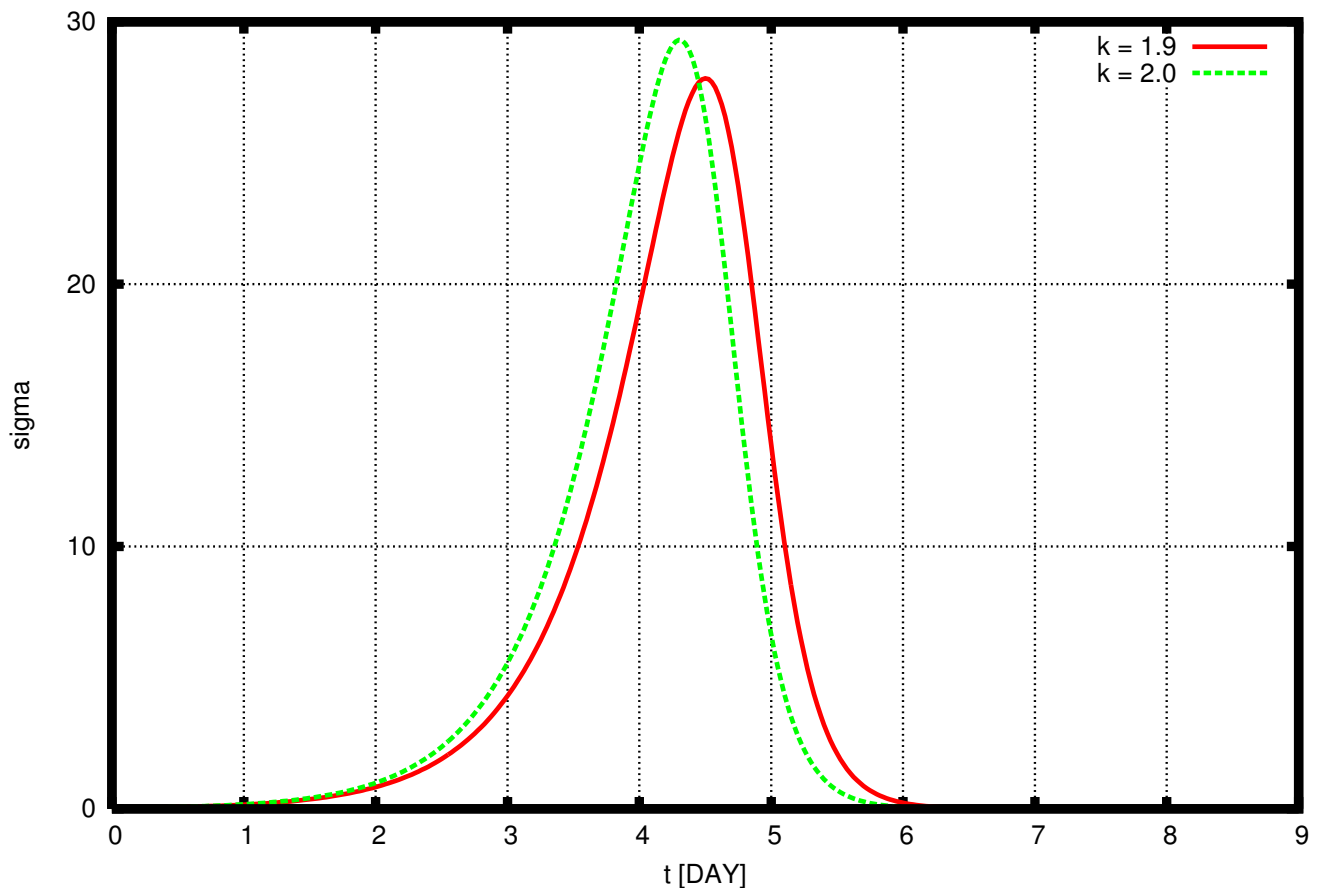

Figure 5: Numerical simulation for growth of microorganism. $\sigma_{0} \approx 0.029827$ and $h=500.0$, and $k=1.9$ and $k=2.0$. 
the primary role in the biodegradation process. The change of population of $S$. terrae according to the residual amount of PEG supports the results shown in Figure 5. Although some details are different between the results shown in Figure 5 and the experimental results for PEG 6000 since the data used in the analysis and simulation originate in a different experiment, the numerical simulation captures the behaviour of the microorganism involved in the biodegradation of PEG.

\section{Discussion}

Previous models involve no information concerning growth of microbial population on polymers. Growth of microbial population was taken into consideration in modelling assuming its logistic growth with degraded monomer units as carbon source. Numerical results show that the model is practically appropriate.

In the environment or in sewer disposal, the time factor should also depend on other factors such as temperature or dissolved oxygen. Once those essentials are incorporated into the time dependent factor, the time dependent exogenous depolymerization model and the techniques based on the model should be applicable to assess biodegradability of xenobiotic polymers.

Acknowledgements: The authors thank Ms Y. Shimizu for her technical support. This work was supported by JSPS KAKENHI 20540118.

\section{References}

[1] F. Kawai, M. Watanabe, M. Shibata, S. Yokoyama, and Y. Sudate. Experimental analysis and numerical simulation for biodegradability of 
polyethylene. Polymer Degradation and Stability, 76:129-135, 2002. doi:10.1016/S0141-3910(02)00006-X C683, C684

[2] F. Kawai, M. Watanabe, M. Shibata, S. Yokoyama, Y. Sudate, and S. Hayashi. Comparative study on biodegradability of polyethylene wax by bacteria and fungi. Polymer Degradation and Stability, 86:105-114, 2004. doi:10.1016/j.polymdegradstab.2004.03.015 C683, C684

[3] Fusako Kawai and Shogo Enokibara. Symbiotic degradation of polyethylene glycol (peg) 20,000-phthalate polyester by phthalate esterand peg 20,000-utilizing bacteria. Journal of Fermentation and Bioengineering, 82(6):575-579, 1996. C690

[4] M. Watanabe and F. Kawai. Numerical simulation of microbial depolymerization process of exogenous type. ANZIAM J., 46(E):C1188-C1204, 2005. Proc. of 12th Computational Techniques and Applications Conference, CTAC-2004, Melbourne, Australia in September 2004, Editors: Rob May and A. J. Roberts http://anziamj . austms.org.au/ojs/index.php/ANZIAMJ/article/view/1014 C683

[5] M. Watanabe and F. Kawai. Mathematical study of the biodegradation of xenobiotic polymers with experimental data introduced into analysis. ANZIAM J., 47:C665-C681, 2007. Proceedings of the 7th Biennial Engineering Mathematics and Applications Conference, EMAC-2005, Melbourne, Editors: Andrew Stacey and Bill Blyth and John Shepherd and A. J. Roberts, http://anziamj.austms.org.au/ojs/index.php/ ANZIAMJ/article/view/1069 C683

[6] M. Watanabe and F. Kawai. Mathematical analysis of microbial depolymerization processes of xenobiotic polymers. In Geoffry N. Mercer and A. J. Roberts, editors, Proceedings of the 14 th Biennial Computational Techniques and Application Conference, CTAC2008, volume 50 of ANZIAM J., pages C930-C946, 2009. http://anziamj. austma.org.au/ojs/index.php/ANZIAMJ/article/view/1465 C683, C687, C688, C689 
[7] M. Watanabe and F. Kawai. Modeling and simulation of biodegradation of xenobiotic polymers based on experimental results. In BIOSIGNALS 2009, Second International Conference on Bio-inspired Systems and Signal Processing, Proceedings, Porto, Portugal, 14-17 January, 2009, pages 25-34. INSTICC Press, 2009. C683

[8] M. Watanabe and F. Kawai. Study on effects of microorganism in depolymerization process of xenobiotic polymers by modeling and simulation. In Ana Fred, Joaquim Filipe, and Hugo Gamboa, editors, Proceedings of the First International Conference on Bioinformatics, Valencia, Spain, January 20-23, 2010, pages 181-186. 2010 INSTICC, Institute for Systems and Technologies of Information, Control and Communication, 2010. C683, C687, C688, C689, C690

[9] M. Watanabe, F. Kawai, M. Shibata, S. Yokoyama, and Y. Sudate. Computational method for analysis of polyethylene biodegradation. Journal of Computational and Applied Mathematics, 161(1):133-144, December 2003. doi:10.1016/S0377-0427(03)0051-X C683, C684

[10] M. Watanabe, F. Kawai, M. Shibata, S. Yokoyama, Y. Sudate, and S. Hayashi. Analytical and computational techniques for exogenous depolymerization of xenobiotic polymers. Mathematical Biosciences, 192:19-37, 2004. doi:10.1016/j.mbs.2004.06.006 C684

\section{Author addresses}

1. M. Watanabe, Graduate School of Environmental Science, Okayama University, Okayama, JAPAN. mailto: watanabe@ems. okayama-u.ac.jp

2. F. Kawai, Center for Nanomaterials and Devices, Kyoto Institute of Technology, Kyoto, JAPAN. 